\title{
Effects of aging on nitrergic system in human basal nuclei
}

\author{
Efeitos do envelhecimento sobre o sistema nitrérgico dos núcleos da base em humanos \\ Bruno Lopes dos Santos
}

Resumo da Dissertação de Mestrado apresentada ao Departamento de Neurociências e Ciências do Comportamento da Faculdade de Medicina de Ribeirão
Preto da Universidade de São Paulo. Área de Neurologia; subárea de Neurologia Clínica. Ribeirão Preto SP, Brasil. Correspondence: Bruno Lopes dos Santos; Departamento de Neurociências e Ciências do Comportamento, Faculdade de Medicina de Ribeirão Preto; Av. Bandeirantes, 3900; 14049-900 Ribeirão Preto SP, Brasil; E-mail: bruls4@usp.br

Orientador: Vitor Tumas.

Coorientadora: Elaine A. Del-Bel.

Received 14 May 2014; Received in final form 27 May 2014; Accepted 23 June 2014.

\begin{abstract}
Nitric oxide (NO) is a gaseous molecule that plays a role in a number of physiologic processes. The available evidence suggests that NO is a major neurotransmitter involved in motor control and emotion/behavior modulation. To investigate the distribution and morphology of the nitrergic system in human basal nuclei, we studied samples from the striatum, globus pallidus, subthalamic nucleus, substantia nigra and pedunculopontine nucleus of 20 human brains from subjects without neurologic/psychiatric diseases. The samples were stained for NADPH-diaphorase using histochemistry and for neuronal NO synthase using immunohistochemistry. We then analyzed the nitrergic neuronal density and its morphometric parameters. Our data demonstrated that: (I) the most posterior regions of the striatum exhibit a higher neuronal density; (II) the limbic cortex-associated areas of the striatum exhibit higher neuronal density than other functional subdivisions; (III) approximately $90 \%$ of the neurons in the subthalamic nucleus express NO; (IV) the pedunculopontine nucleus exhibits a massive nitrergic neuronal density; $(V)$ in the globus pallidus, there is a marked presence of NO neurons in the medial medullary lamina; and $(\mathrm{VI})$ nitrergic neurons were not detected in the substantia nigra. Aging did not change the neuronal density or the morphometric parameters of nitrergic neurons in the analyzed nuclei.
\end{abstract}

Keywords: nitric oxide, basal nuclei, striatum, subthalamic nucleus, aging. 